\title{
Spatially Targeted Communication and Self-Assembly
}

\author{
Nithin Mathews ${ }^{1}$, Anders Lyhne Christensen ${ }^{2}$, Rehan O'Grady ${ }^{1}$, and Marco Dorigo ${ }^{1}$
}

\begin{abstract}
We introduce spatially targeted communication a communication method for multirobot systems. This method allows an individual message sending robot to isolate selected message recipient robots based on their spatial location. The recipient robots can then be sent information targeted solely at them, even if the sending robot uses a broadcast communication modality. We demonstrate spatially targeted communication using a heterogeneous multirobot system composed of flying robots and ground-based self-assembling robots. Flying robots use their privileged view of the environment to determine and communicate information to groups of ground-based robots on what morphologies to form to carry out upcoming tasks.
\end{abstract}

\section{INTRODUCTION}

Multirobot systems have a number of advantages over single robot systems including the ability to execute multiple tasks in parallel. In multirobot systems, robots also have the potential to cooperate if a task is too difficult for a single robot to solve alone. Self-assembly - a reversible process by which the robots autonomously form physical connections with one another [1] — enables tightly integrated physical cooperation between robots. We use self-assembling robots built as part of the EU-funded Swarmanoid project [2] that are capable of forming a large number of morphologies of different shapes and sizes, see Fig. 1 for examples.

The environmental perception of ground-based robots is often limited, partly due to the limitations of the sensory hardware and partly due to the vantage point of ground-based robots. From the perspective of a self-assembling robot, it can be difficult to perceive and estimate the parameters of tasks with sufficient completeness and accuracy to deduce which (if any) morphology could be formed to solve the task. This is illustrated in Fig. 2(a), where a group of selfassembling robots are approaching a hill. In Fig. 2(b), the limited view of a self-assembling robot approaching the hill is shown. A flying robot, on the other hand, can get a good overview of the task and of the robots on the ground from an elevated position. Figure 2(c) is an image captured by a flying robot's camera. Using the information collected from its vantage point, the flying robot can run on-board simulations to find a morphology that the self-assembling robots can form to get over the hill - an essential analysis that the self-assembling robots would have been incapable of by themselves. The range of tasks that can be solved by selfassembling robots can thus be extended through cooperation with flying robots.

\footnotetext{
${ }^{1}$ IRIDIA-CoDE, Université Libre de Bruxelles, Avenue Franklin Roosevelt 50, 1050 Brussels, Belgium \{nmathews, rogrady, mdorigo\}eulb.ac.be

${ }^{2}$ Instituto de Telecomunicações \& Instituto Universitário de Lisboa (ISCTE-IUL), Lisbon, Portugal anders.christensen discte.pt
}

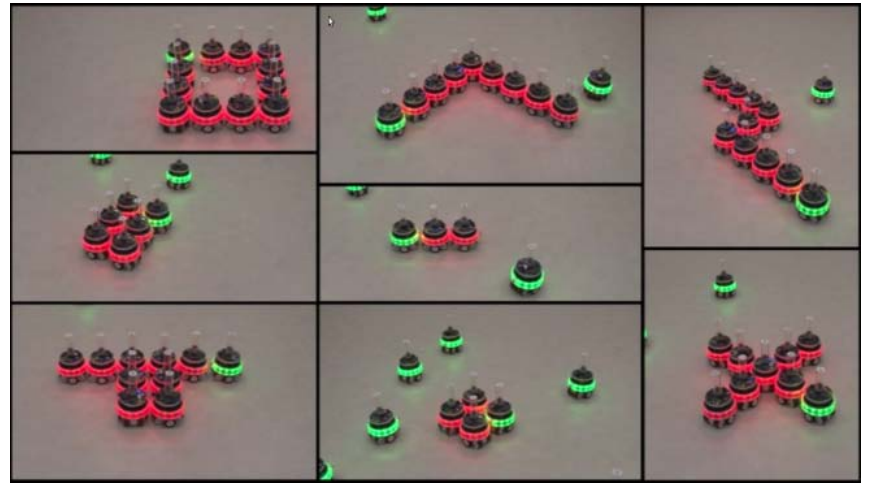

Fig. 1: Example morphologies that can be formed by the self-assembling robots used in this study.

Communication plays a key role in enabling cooperation in heterogeneous multirobot systems composed of flying robots and ground-based robots. In such systems, flying robots often need to give instructions to particular groups of robots on the ground. In this study, a flying robot that has detected an object of interest on the other side of a steep hill needs to communicate self-assembly instructions to the four robots located closest to the hill - to self-assemble into a line morphology and to cross the hill. Such selective communication is non-trivial, as flying robots and ground robots have such different views of the world (see Fig. 2(b) and Fig. 2(c)) that it is hard for them to agree on a common frame of reference.

\section{COMMUNICATION AND COOPERATION}

We proposed spatially targeted communication [3] as a method to allow a single robot to establish a communication link with one or more co-located robots using broadcast messages. In this study, we present an experiment in which a flying robot estimates the parameters of an upcoming task on the ground by computing height maps of the ground based on stereo images. These parameters are then used in onboard simulations that are executed to find a morphology appropriate for the task [4]. The flying robot then establishes a communication link with an appropriate number of robots on the ground, and sends the group instructions on how to form the morphology [5]. Finally, the self-assembling robots form the morphology using a recruitment and guidance-based approach [6] and carry out the task.

It should be noted that our approach relies only on standard LEDs and cameras and does not require any specialized hardware. Furthermore, our approach does not require a global map of the environment, robots IDs, GPS, or any 


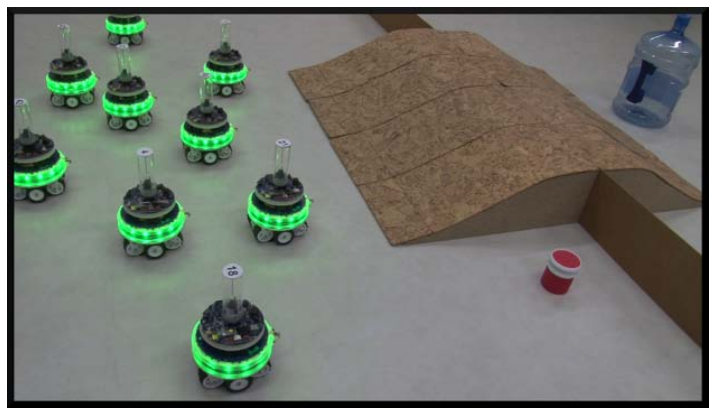

(a)

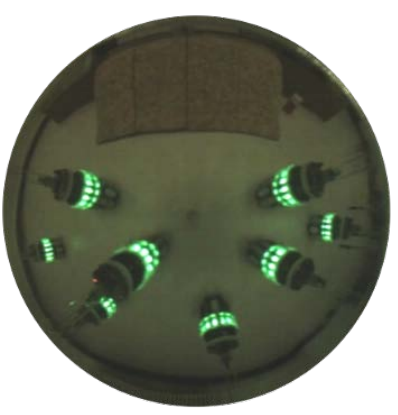

(b)

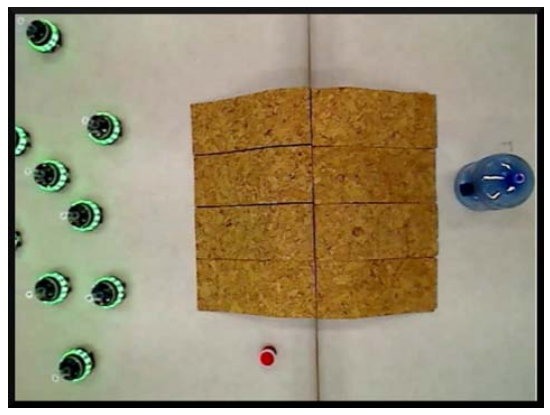

(c)

Fig. 2: Self-assembling robots approaching a hill as seen from different perspectives: (a) from an external video camera on a tripod, (b) by a ground-based robot using its omni-directional camera, and (c) from a flying robot located above the hill.

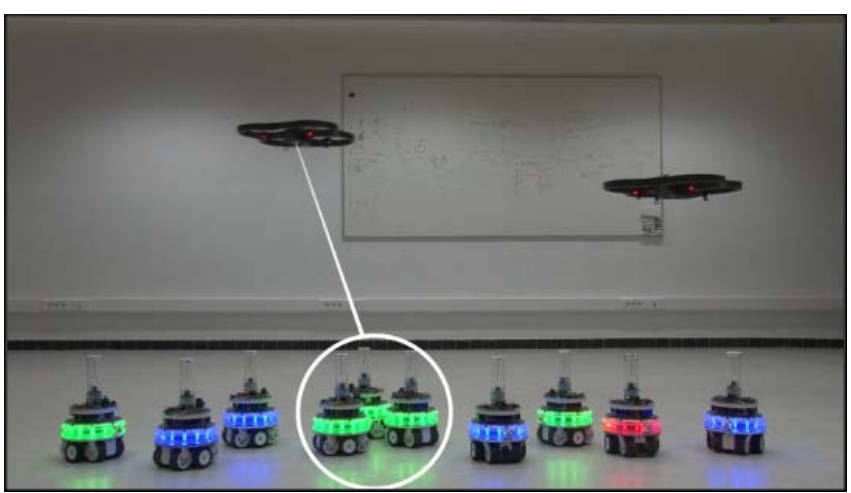

Fig. 3: A schematic highlighting spatially targeted communication. The overlay shows the subgroup of robots with which the flying robot has the intention to communicate.

external robot tracking infrastructure. Spatially targeted communication is therefore ideally suited to swarms of robots and can be used outside of a laboratory environment.

\section{RESULTS}

The accompanying video shows how spatially targeted communication can be used to enable cooperation between flying robots and ground-based robots. In particular, we show how cooperation with flying robots can benefit ground-based self-asembling robots in their quest to carry out tasks that are too difficult for a single robot to solve alone. In the video, we explain when and why cooperation is necessary (see for example, Fig. 3 in which one of the flying robots needs to communicate to three specific robots on the ground). We then give details of our approach and show how it works. We go on to demonstrate how spatially targeted communication can enhance the capabilities of self-assembling robots in real world task-execution scenarios.

\section{ACKNOWLEDGMENTS}

The research leading to the results presented in this study has received funding from the European Research Council under the European Union's Seventh Framework Programme (FP7/2007-2013) / ERC grant agreement $\mathrm{n}^{\circ}$ 246939. Marco Dorigo and Rehan O’Grady acknowledge support
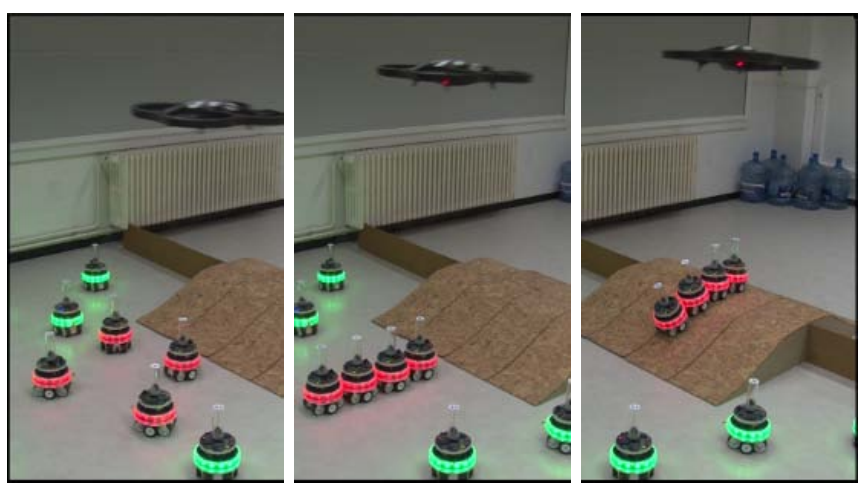

Fig. 4: Three frames from the accompanying video: a flying robot instructs a group of robots on the ground how to safely cross a hill by self-assembling into a linear morphology.

from the Fund for Scientific Research F.R.S.-FNRS of Belgium's French Community, of which they are a research director and a postdoctoral researcher, respectively. This work was also partially supported by FCT grant PTDC/EEACRO/104658/2008 and PEst-OE/EEI/LA0008/2011.

\section{REFERENCES}

[1] G. M. Whitesides and B. Grzybowski, "Self-assembly at all scales," Science, vol. 295, no. 5564, pp. 2418-2421, 2002.

[2] M. Dorigo et al., "Swarmanoid: a novel concept for the study of heterogeneous robotic swarms," IEEE Robotics \& Automation Magazine, in press, 2012.

[3] N. Mathews, A. L. Christensen, E. Ferrante, R. O'Grady, and M. Dorigo, "Establishing spatially targeted communication in a heterogeneous robot swarm," in Proceedings of 1th International Conference on Autonomous Agents and Multiagent Systems (AAMAS 2012). IFAAMAS, Richland, SC, 2010, pp. 939-946.

[4] N. Mathews, A. Stranieri, A. Scheidler, and M. Dorigo, "Supervised morphogenesis - morphology control of ground-based self-assembling robots by aerial robots," in Proceedings of 11th International Conference on Autonomous Agents and Multiagent Systems (AAMAS 2012). IFAAMAS, Richland, SC, 2012, pp. 97-104.

[5] A. L. Christensen, R. O'Grady, and M. Dorigo, "SWARMORPH-script: a language for arbitrary morphology generation in self-assembling robots," Swarm Intelligence, vol. 2, no. 2-4, pp. 143-165, 2008.

[6] N. Mathews, A. L. Christensen, R. O’Grady, P. Rétornaz, M. Bonani, F. Mondada, and M. Dorigo, "Enhanced directional self-assembly based on active recruitment and guidance," in Proceedings of the 2011 IEEE/RSJ International Conference on Intelligent Robots and Systems. IEEE Computer Society Press, Los Alamitos, CA, 2011, pp. 4762-4769. 